\title{
Tailoring Imprinted Titania Nanoparticles for Purines Recognition
}

\author{
Adnan Mujahid, ${ }^{1}$ Amna Najeeb, ${ }^{1}$ Aimen Idrees Khan, ${ }^{1}$ Tajamal Hussain, ${ }^{1}$ \\ Muhammad Hamid Raza, ${ }^{1}$ Asma Tufail Shah, ${ }^{2}$ Naseer Iqbal, ${ }^{2}$ and Mirza Nadeem Ahmad \\ ${ }^{1}$ Institute of Chemistry, University of the Punjab, Quaid-i-Azam Campus, Lahore 54590, Pakistan \\ ${ }^{2}$ Interdisciplinary Research Centre for Biomedical Materials, COMSATS Institute of Information Technology, Defence Road, \\ Off Raiwind Road, Lahore 54000, Pakistan \\ ${ }^{3}$ Institute of Chemistry, Government College University, Faisalabad 38030, Pakistan
}

Correspondence should be addressed to Adnan Mujahid; adnanmujahid.chem@pu.edu.pk

Received 7 June 2015; Accepted 21 July 2015

Academic Editor: Kourosh Kalantar-Zadeh

Copyright (c) 2015 Adnan Mujahid et al. This is an open access article distributed under the Creative Commons Attribution License, which permits unrestricted use, distribution, and reproduction in any medium, provided the original work is properly cited.

\begin{abstract}
Molecular imprinted titania nanoparticles were developed for selective recognition of purines, for example, guanine and its final oxidation product uric acid. Titania nanoparticles were prepared by hydrolysis of titanium butoxide as precursor in the presence of pattern molecules. The morphology of synthesized nanoparticles is evaluated by SEM images. Recognition characteristics of imprinted titania nanoparticles are studied by exposing them to standard solution of guanine and uric acid, respectively. The resultant change in their concentration is determined by UV/Vis analysis that indicated imprinted titania nanoparticles possess high affinity for print molecules. In both cases, nonimprinted titania is taken as control to observe nonspecific binding interactions. Cross sensitivity studies suggested that imprinted titania is at least five times more selective for binding print molecules than competing analyte thus indicating its potential for bioassay of purines.
\end{abstract}

\section{Introduction}

Molecular imprinting technology (MIT) is getting increasing attention of scientific community owing to its numerous applications [1] in various fields such as catalysis [2], chiral separations [3], chemical sensor design [4], advance drug delivery systems [5], process control [6], and many others. Among all, the crafting of synthetic receptors is highly valuable in order to design low cost biomimetic materials $[7,8]$ since natural antibodies are very expensive and cannot be reused. The recognition characteristics can be introduced in various materials ranging from bulk polymers to thin films and even in nanomaterials. MIT is one of the most promising approaches to design synthetic receptor materials as molecular imprinted polymers offer both chemical and geometrical fitting [9] to target molecules. As in imprinted polymers, the functional monomers undergo self-organization to develop noncovalent interactions with template. Once the polymerization is completed, polymer chains are fixed around the template molecules which can be washed out by treating polymer with suitable solvent. The washed polymer possesses adapted interaction centers complementary to the shape and dimensions of template; thus, it offers the geometrical fitting for target analyte [10]. In addition, the analyte can also be recognized through different chemical forces mainly noncovalent ones, for example, hydrogen bonding and others which accomplish chemical fitting. Previously, the major concern in using bulk imprinted polymeric materials was inhomogeneous particles of large size due to mechanical crushing and grindings. This often leads to small recoverable mass of imprinted particles and less efficient bindings. In order to avoid such problems the concept of nanotechnology was integrated with molecular imprinting $[11,12]$ to produce much finer, homogenous, and evenly distributed particles with suitable recognition characteristics. Therefore, in recent years the focus has been shifted from bulk imprinted polymers to nanosized particles that offer high surface area with promising sensing $[13,14]$ potential. 
TABLE 1: FTIR data of as-prepared guanine imprinted titania nanoparticles.

\begin{tabular}{lccccc}
\hline Functional units & Ti-O & C-N & N-H bending & N-H stretching & C=N \\
\hline Absorbance frequency $\left(\mathrm{cm}^{-1}\right)$ & $600-800$ & 1371 & 1535 & $3100-3500$ & 1691 \\
\hline
\end{tabular}

In present work, imprinted titania nanoparticles were synthesized for recognition of purine base, that is, guanine and its oxidation product uric acid. Guanine is a nucleobase and has vital role in storing genetic information whereas uric acid is an end product of purine breakdown and its elevated level in blood could lead to gout and other related problems. Although the nature and functions of these two compounds are different as one is the end product of the other, their detection is important in many perspectives. The synthesized particles were characterized with microscopic and spectroscopic techniques to investigate their recognition performance.

\section{Materials and Methods}

2.1. Chemical and Reagents. All the chemicals and reagents used in this study were obtained from Sigma Aldrich and used without prior treatment.

2.2. Characterization. The imprinting of template in titania nanoparticles and its subsequent removal were studied by Labomed UVD-2950 spectrophotometer. Guanine imprinted titania nanoparticles were characterized by Bruker FTIR Vector 22 to study template functional units in nanoparticles matrix. The particle size and morphology of titania nanoparticles were examined by Hitachi S-4700 scanning electron microscope (SEM) images.

2.3. Synthesis of Imprinted Titania Nanoparticles. Guanine imprinted titania nanoparticles were developed following already reported method [15] with slight modification. Firstly, $0.284 \mathrm{~g}$ of titanium butoxide was dissolved in $5 \mathrm{~mL}$ of isopropanol and subjected under constant stirring at $60^{\circ} \mathrm{C}$. Now, $0.005 \mathrm{~g}$ of guanine was dissolved in $5 \mathrm{~mL}$ acidic solvent mixture, that is, methanol and $0.1 \mathrm{~N} \mathrm{HCl}$ in $60: 40$ ratio, respectively. After adding template solution, the nanoparticles started to form due to acid catalyzed hydrolysis. The reaction was continued for one hour until no further precipitation occurred on adding acidic solvent. The reaction mixture was centrifuged at $5000 \mathrm{rpm}$ for 10 minutes to separate the synthesized particles. Similar procedure was followed for synthesizing uric acid imprinted nanoparticles; however, here basic solvent, that is, $0.1 \mathrm{~N} \mathrm{NaOH}$, was used keeping the rest of the procedure and conditions exactly the same. The nonimprinted titania nanoparticles were prepared in the same manner without adding print molecules.

2.4. Washing and Rebinding Studies. The two different batches of synthesized nanoparticles, that is, guanine imprinted and uric acid imprinted titania nanoparticles, were washed with water and methanol solvent system comprising equal volume to remove print molecules. The supernatant of washed particles was analyzed by UV/Vis in the range of
200-400 $\mathrm{nm}$ to determine the residual concentration of print molecules in titania nanoparticles. Washing process was continued until no appreciable absorbance of guanine and uric acid was observed in supernatants. These nanoparticles were dried in oven to remove solvent and stored for further studies.

For rebinding studies, $20 \mathrm{mg}$ of guanine imprinted nanoparticles was treated with $20 \mathrm{~mL}$ of standard guanine solution and stirred for one hour at ambient conditions. After that, the mixture was centrifuged and supernatant was subjected to $\mathrm{UV} / \mathrm{Vis}$ analysis for calculating the concentration of leftover template. The difference in absorbance of guanine solution before and after exposing with imprinted titania nanoparticles gives the quantitative information about the amount of print molecules binding. A similar procedure was repeated for uric acid imprinted titania nanoparticles.

\section{Results and Discussion}

As-prepared molecular imprinted titania nanoparticles were washed with water and methanol solvent system to release print molecules from titania network and also remove unwanted reaction species. The supernatant of washed nanoparticles was analyzed by UV/Vis in the range of $200-400 \mathrm{~nm}$ to observe the absorbance of released print molecules. Both guanine and uric acid imprinted titania nanoparticles were subjected to washing phases separately and corresponding decrease in the absorbance of supernatants was monitored. It was observed that after five washing phases the absorbance of both guanine and uric acid in respective supernatants approaches zero. The result of guanine and uric acid washing phases against their decreasing absorbance is shown in Figure 1.

Guanine imprinted titania nanoparticles were also characterized by FTIR to observe template functional groups in nanoparticles matrix. FTIR data of imprinted nanoparticles has been summarized in Table 1 . The formation of titania network was noticed by broad absorption band of Ti-O functional units in the range of $600-800 \mathrm{~cm}^{-1}$. As guanine is taken as model template for imprinting, a broad and strong absorption band due to stretching vibrations of $\mathrm{N}-\mathrm{H}$ groups is observed at $3100-3500 \mathrm{~cm}^{-1}$ which indicates its association with terminal $\mathrm{O}-\mathrm{H}$ groups of titania nanoparticles through hydrogen bonding interactions. Furthermore, N-H bending vibrations were observed near $1535 \mathrm{~cm}^{-1}$. The stretching vibrations of other functional groups in guanine such as $\mathrm{C}-\mathrm{N}$ and $\mathrm{C}=\mathrm{N}$ were noticed at $1371 \mathrm{~cm}^{-1}$ and $1691 \mathrm{~cm}^{-1}$, respectively.

Surface characterization of synthesized titania nanoparticles was carried out by SEM; additionally, a 3D image of this surface was also developed as shown in Figure 2(b). These images reveal the morphological features of imprinted titania nanoparticles indicating homogenous dispersion which is 


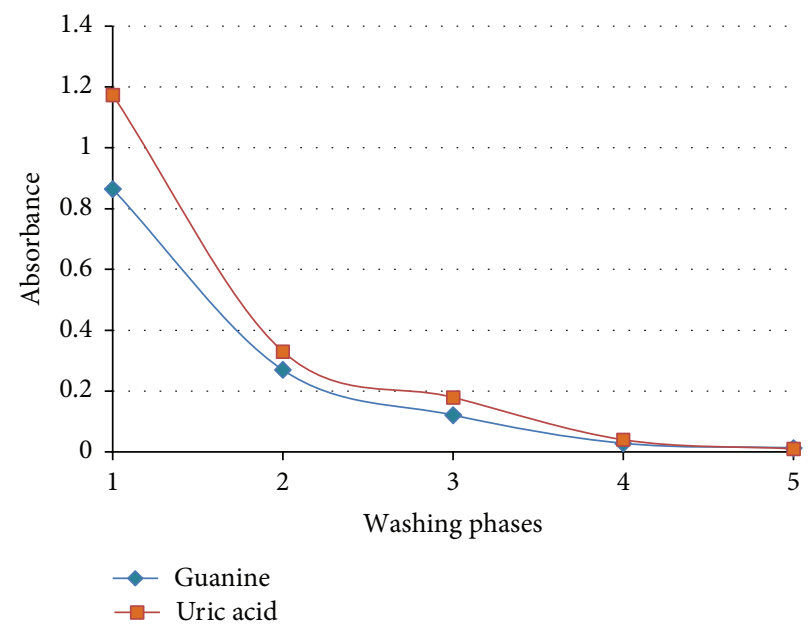

FIgURE 1: Removal of print molecules on washing guanine and uric acid imprinted titania nanoparticles.

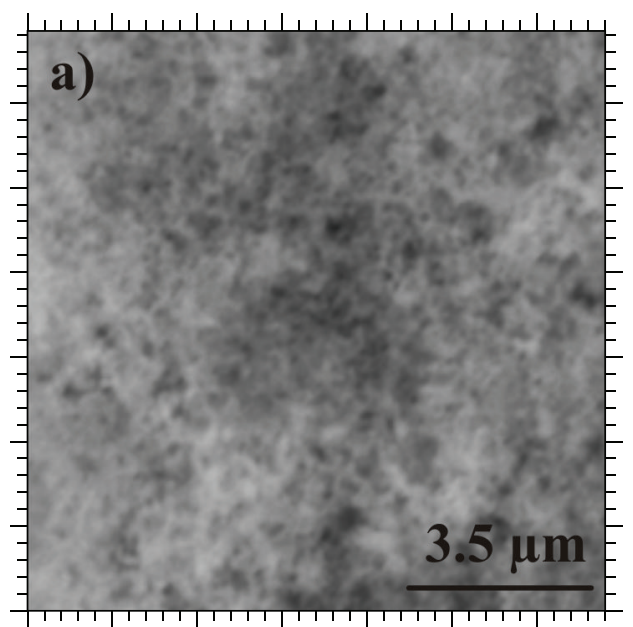

(a)

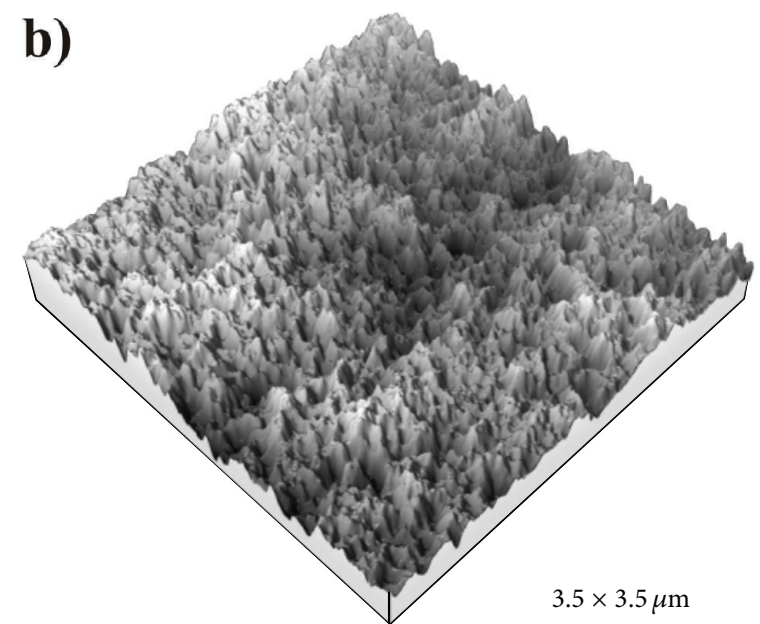

(b)

FIGURE 2: SEM surface images of imprinted titania nanoparticles.

advantageous for uniform distribution of adapted interaction centers in titania network. This ensures high binding affinity due to large number of interaction sites.

The binding affinity of imprinted titania nanoparticles was compared to nonimprinted titania. For instance, the absorbance of standard guanine solution, for example, $1 \mathrm{mmol}$, was monitored before and after treating with imprinted nanoparticles. Same practice was followed in case of nonimprinted titania nanoparticles. The change in absorbance is taken as directly related to guanine adsorption by titania nanoparticles, as the higher the change in absorbance, the more the guanine bounded by nanoparticles. The relative change in absorbance for guanine imprinted and nonimprinted titania nanoparticles is shown in Figure 3. It can be seen that the change in absorbance for imprinted titania is about seven times higher as compared to nonimprinted titania nanoparticles. This clearly indicates that imprinted titania possesses tailored recognition centers by which it interacts with target

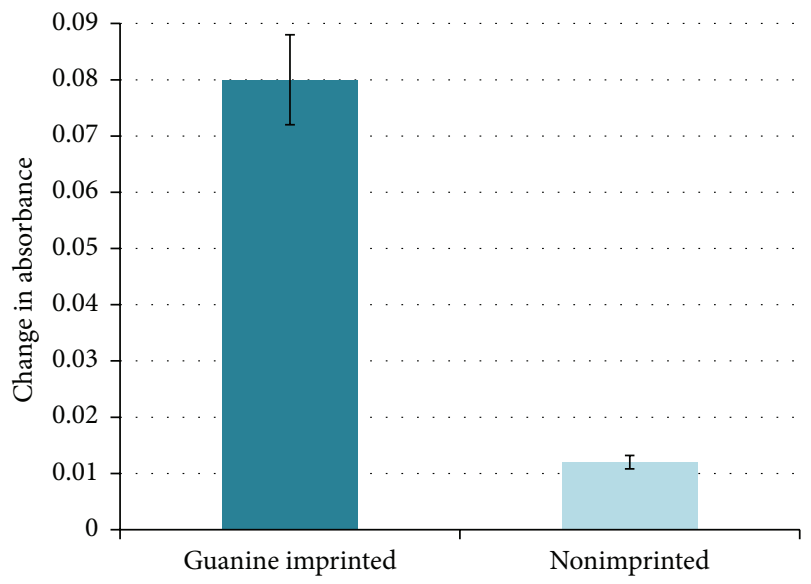

FIGURE 3: Comparison of relative change in absorbance for guanine imprinted and nonimprinted titania nanoparticles when exposed to $1 \mathrm{mmol}$ guanine solution. 


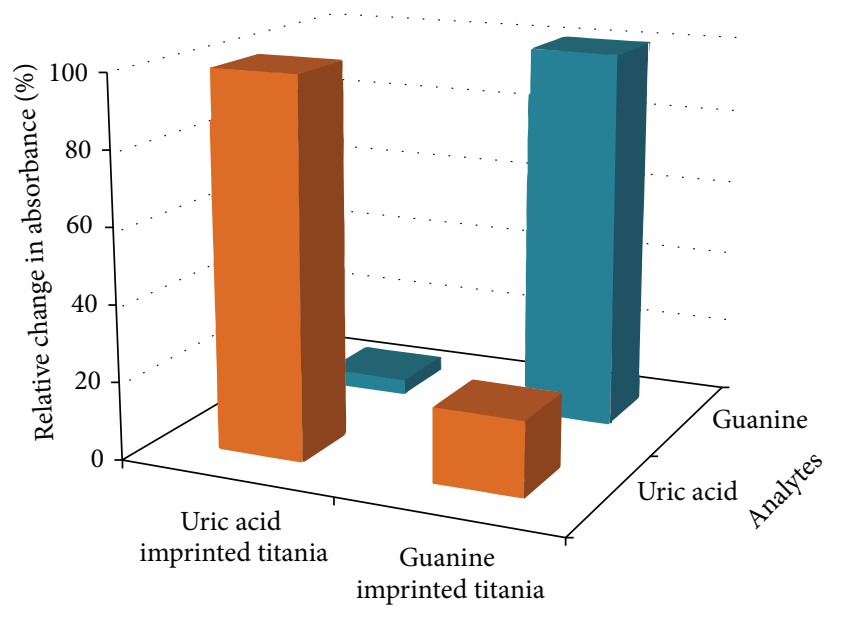

FIGURE 4: Relative change in absorbance for guanine and uric acid imprinted titania nanoparticles when treated against equimolar solutions of guanine and uric acid separately.

analytes whereas nonimprinted matrix does not contain such binding sites. Furthermore, the nonimprinted material can be taken as control and in this way all the nonspecific interactions and bindings can be compared. This bar graph in Figure 3 clearly demonstrates successful imprinting of guanine in titania nanonetwork. In case of uric acid, the shift in absorbance for imprinted titania was six times higher than nonimprinted titania.

Apart from testing the affinity of imprinted titania nanoparticles for template analytes, that is, guanine and uric acid, the selectivity of both types of imprinted nanoparticles was also evaluated on exposing competing bioanalyte. Molecular imprinted materials developed for guanine recognition in earlier report [16] had shown good sensitivity and selectivity for guanine; however, uric acid interactions with imprinted matrix were not studied. This is important in view of guanine and uric acid existence in complex biological fluid such as blood serum and also as one biomolecule is the oxidation product of the other. For this purpose, the change in absorbance for guanine imprinted titania nanoparticles was monitored on treating against guanine and uric acid solutions separately at equimolar concentrations. And in the same way the change in absorbance for uric acid imprinted titania particles was calculated. The normalized change in absorbance for both types of nanoparticles is highlighted in Figure 4.

From this graph, it is evident that both guanine and uric acid imprinted titania nanoparticles prefer to bind their respective print molecules as compared to other bioanalytes. This suggests that imprinted titania nanoparticles contain highly adapted interaction sites which are complementary in geometrical and chemical functionality of print molecules. The tailored centers recognize target analytes through various types of chemical interactions, for example, hydrogen bonding, van der Waal forces, and others resulting in enhanced selectivity. Furthermore, it is also plausible that the same type of nanoparticles can be tuned accordingly for recognition of desired analytes by molecular imprinting strategy.

\section{Conclusion}

Tailored recognition properties can be introduced in nanomaterials using molecular imprinting procedures. In this study, the developed molecular imprinted titania nanoparticles have shown enhanced affinity for target bioanalytes as compared to nonimprinted ones; furthermore, they exhibited substantially high selectivity for imprint molecules. This indicates the potential of titania nanoparticles for bioassays of diverse analytes. Furthermore, titania being robust material can tolerate harsh environments and therefore could possibly be used for working in complex biological matrices.

\section{Conflict of Interests}

The authors declare that there is no conflict of interests regarding the publication of this paper.

\section{References}

[1] L. I. Andersson, "Molecular imprinting: developments and applications in the analytical chemistry field," Journal of Chromatography B: Biomedical Sciences and Applications, vol. 745, no. 1, pp. 3-13, 2000.

[2] G. Wulff and J. Liu, "Design of biomimetic catalysts by molecular imprinting in synthetic polymers: the role of transition state stabilization.", Accounts of Chemical Research, vol. 45, no. 2, pp. 239-247, 2012.

[3] W. J. Cheong, S. H. Yang, and F. Ali, "Molecular imprinted polymers for separation science: a review of reviews," Journal of Separation Science, vol. 36, no. 3, pp. 609-628, 2013.

[4] A. Mujahid, P. A. Lieberzeit, and F. L. Dickert, "Chemical sensors based on molecularly imprinted sol-gel materials," Materials, vol. 3, no. 4, pp. 2196-2217, 2010.

[5] B. Sellergren and C. J. Allender, "Molecularly imprinted polymers: a bridge to advanced drug delivery," Advanced Drug Delivery Reviews, vol. 57, no. 12, pp. 1733-1741, 2005.

[6] P. A. Lieberzeit, A. Afzal, D. Podlipna, S. Krassnig, H. Blumenstock, and F. L. Dickert, "Printing materials in micro- and nano-scale: systems for process control," Sensors and Actuators B: Chemical, vol. 126, no. 1, pp. 153-158, 2007.

[7] R. Schirhagl, U. Latif, D. Podlipna, H. Blumenstock, and F. L. Dickert, "Natural and biomimetic materials for the detection of insulin," Analytical Chemistry, vol. 84, no. 9, pp. 3908-3913, 2012.

[8] A. Mujahid, N. Iqbal, and A. Afzal, "Bioimprinting strategies: from soft lithography to biomimetic sensors and beyond," Biotechnology Advances, vol. 31, no. 8, pp. 1435-1447, 2013.

[9] M. Jenik, A. Seifner, S. Krassnig et al., "Sensors for bioanalytes by imprinting - polymers mimicking both biological receptors and the corresponding bioparticles," Biosensors and Bioelectronics, vol. 25, no. 1, pp. 9-14, 2009.

[10] O. Hayden, K.-J. Mann, S. Krassnig, and F. L. Dickert, "Biomimetic ABO blood-group typing," Angewandte Chemie, vol. 45, no. 16, pp. 2626-2629, 2006.

[11] M. Irshad, N. Iqbal, A. Mujahid et al., "Molecularly imprinted nanomaterials for sensor applications," Nanomaterials, vol. 3, no. 4, pp. 615-637, 2013.

[12] K. Flavin and M. Resmini, "Imprinted nanomaterials: a new class of synthetic receptors," Analytical and Bioanalytical Chemistry, vol. 393, no. 2, pp. 437-444, 2009. 
[13] J. Huang, X. Zhang, Q. Lin et al., "Electrochemical sensor based on imprinted sol-gel and nanomaterials for sensitive determination of bisphenol A," Food Control, vol. 22, no. 5, pp. 786791, 2011.

[14] J. Huang, X. Zhang, S. Liu et al., "Development of molecularly imprinted electrochemical sensor with titanium oxide and gold nanomaterials enhanced technique for determination of 4nonylphenol," Sensors and Actuators B: Chemical, vol. 152, no. 2, pp. 292-298, 2011.

[15] A. Mujahid, A. Khan, A. Afzal et al., "Molecularly imprinted titania nanoparticles for selective recognition and assay of uric acid," Applied Nanoscience, vol. 5, no. 5, pp. 527-534, 2015.

[16] S. E. Diltemiz, A. Denizli, A. Ersöz, and R. Say, "Molecularly imprinted ligand-exchange recognition assay of DNA by SPR system using guanosine and guanine recognition sites of DNA," Sensors and Actuators B: Chemical, vol. 133, no. 2, pp. 484-488, 2008. 

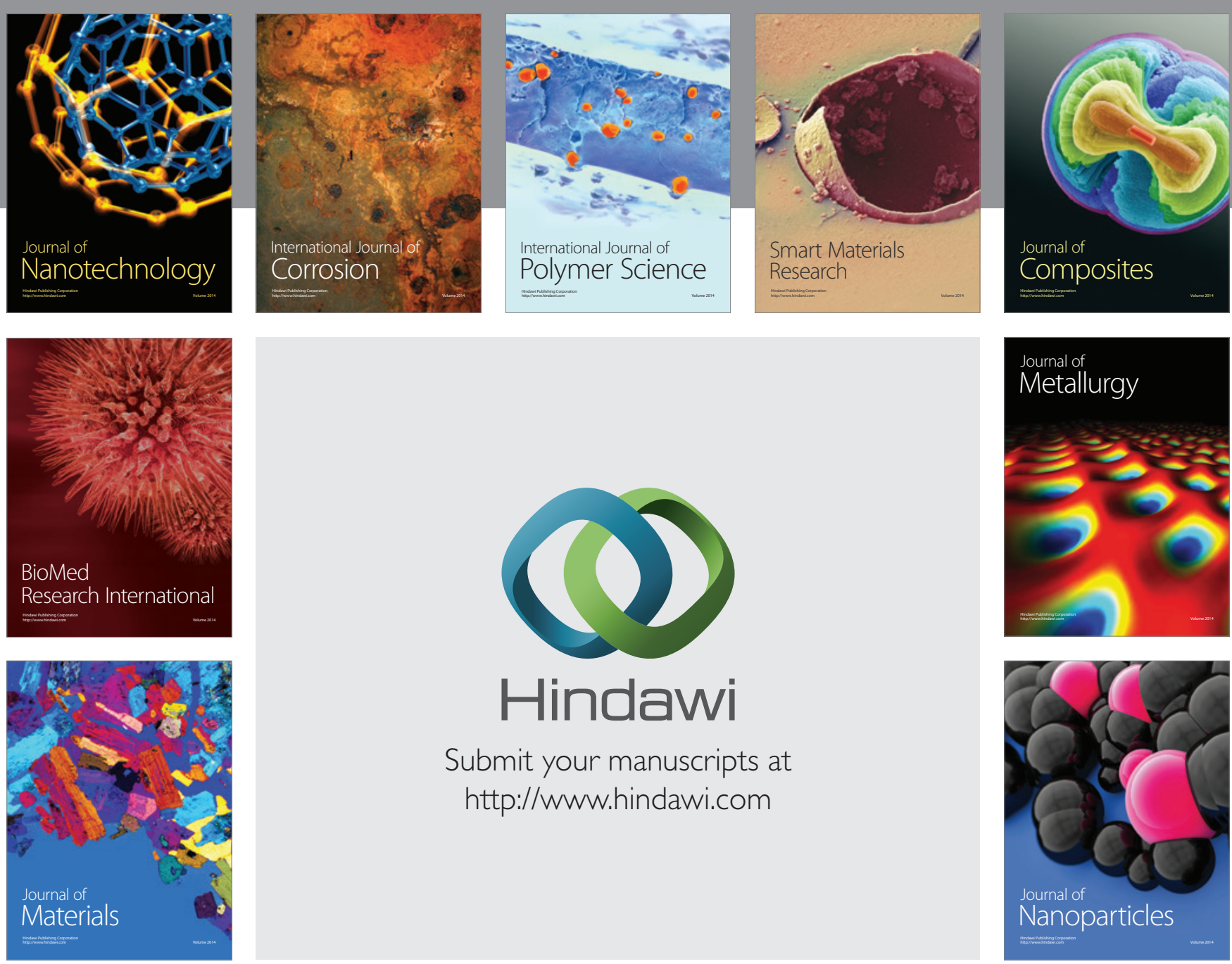

Submit your manuscripts at http://www.hindawi.com
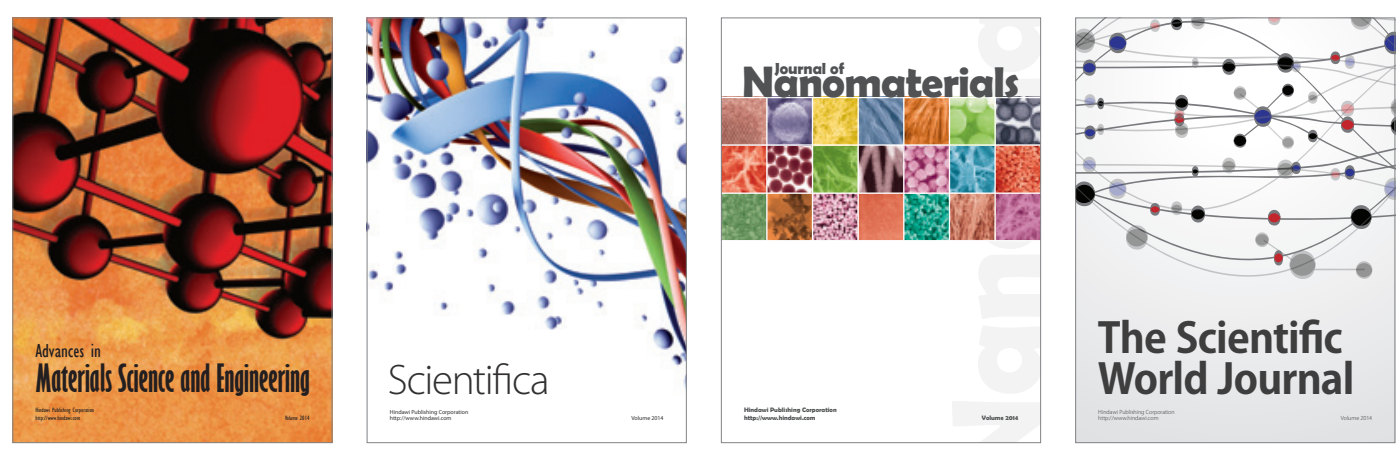

\section{The Scientific World Journal}
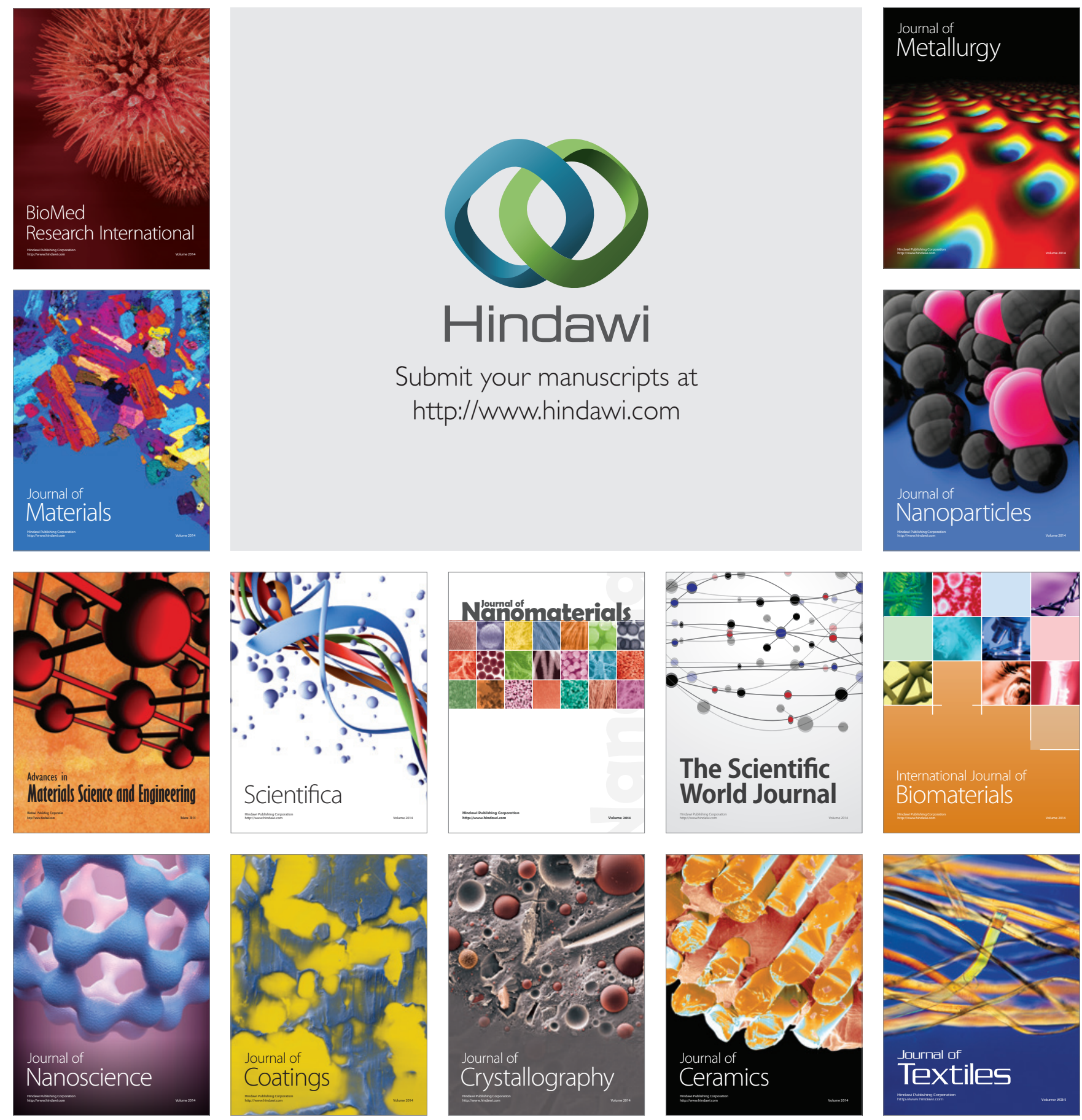